Relations industrielles

Industrial Relations

\title{
Le directeur de personnel
}

\section{T. Roger McLagan}

Volume 2, numéro 9, mai 1947

URI : https://id.erudit.org/iderudit/1023881ar

DOI : https://doi.org/10.7202/1023881ar

Aller au sommaire du numéro

Éditeur(s)

Département des relations industrielles de l’Université Laval

ISSN

0034-379X (imprimé)

1703-8138 (numérique)

Découvrir la revue

Citer cet article

McLagan, T. (1947). Le directeur de personnel. Relations industrielles /

Industrial Relations, 2(9), 4-5. https://doi.org/10.7202/1023881ar

Tous droits réservés (C Département des relations industrielles de l’Université Laval, 1947
Ce document est protégé par la loi sur le droit d'auteur. L'utilisation des services d'Érudit (y compris la reproduction) est assujettie à sa politique d'utilisation que vous pouvez consulter en ligne.

https://apropos.erudit.org/fr/usagers/politique-dutilisation/ 


\section{Les fonctions du directeur de personnel}

Le premier principe qui doit guider l'action d'un directeur de personnel, c'est celui de la justice. On ne doit pas oublier qu'il y a 1ci deux parties en caltse: l'employe et l'entreprise. Le directeur de personnel a une double personnalité: lorsqu'il traite avec la direction de l'établissement, il doit prendre la part du salarié et lorsqu'il traite avec les employés, il doit représenter les intérêts de l'entreprise. Malgré que cette situation puisse sembler singulière il doit en être ainsi afin que le directeur de personnel remplisse efficacement sa fonction.

Le directeur de personnel doit penser en termes de coût de production. Dans l'accomplissement de sa tâche il ne doit pas perdre de vue les fonctions principales de l'entreprise qui se résument à l'emploi de main-d'oeuvre et la réalisation d'un profit. Il doit porter son attention sur le coût de production tout comme le contremaitre à l'usine; il doit même s'en préoccuper davantage puisque sa fonction le place à un poste supérieur.

Parce que ses fonctions en font un homme responsable, le directeur de personnel, à mon avis, doit relever du président du conseil d'administration. Il n'est pas un officier ordinaire qui reçoit ses directives d'un autre; ses attributions, difficiles de délimitation, l'obligent à beaucoup d'initiative. Aussi importe-t-il que l'entreprise accorde une attention de plus en plus marquée aux fonctions du service de personnel. Il est aussi nécessaire de s'occuper des relations humaines que des relations publiques.

La responsabilité de la propagande et de la publicité intérieures incombe au directeur de personnel. Dans une grande entreprise où l'on rencontre un contingent nombreux d'êtres humains, il est indispensable que quelqu'un s'occupe attentivement de la publicité, afin que les employés soient informés de ce qui s'y passe, en vue de pouvoir, le cas échéant, ne pas se laisser influencer par une propagande insidieuse.

\section{Les registres}

L'exécution du travail confié au directeur de personnel, exige la tenue de registres complets d'employés. Une entreprise ne peut être conduite sans l'aide d'états appropriés sur les prix de revient; de même est-il impossible de diriger un service de personnel sans un système adéquat de registres d'employés. Je ne saurais trop insister sur ce point, tellement il a été négligé en ces dernières années.

Il doit y avoir une politique bien définie à l'égard de tels registres et cette politique doit s'inspirer du système conventionnel de comptabilité. On doit poser en principe qu'à compter du jour où un employé est embauché jusqu'au moment de son départ, un système approprié de registres doit contenir fidèlement tout ce qui s'y rapporte. On doit aussi conserver dans un livre journal l'inscription des noms des employés embauchès, congédiés et transiérés.

Une fiche doit être faite au nom de tout nouvel employé et classée au bureau. On doit aussi pourvoir à des formules d'embauchage, de congédiement, de changement de taux, de transfert, etc., d'employés et l'histoire complète de chacun doit apparaître sur une fiche à cette fin. Au départ définitif d'un employé, ses fiches doivent être transférées aux archives et classées selon l'ordre alphabétique. De cette façon, s'il devait revenir plus tard, le

\section{LE DIRECTEUR}

service de placement aurait l'histoire complète de son stage à l'usme et advenant son retour au travail, ses fiches n'auraient qu'à être versees aux dossiers courants.

Les formules destinées au service de placement doivent viser surtout à l'efficacité. C'est pourquoi elles doivent être conçues de façon qu'elles puissent servir à de multiples usages tout en étant peu nombreuses. La tenue soignée d'un fichier au service de placement relève du directeur de personnel.

\section{L'embauchage et le congédiement des employés}

La responsabilité de l'embauchage et du congédiement des employés doit reposer totalement sur le directeur de personnel. Quand les contremaitres requièrent de nouveaux employés, ils doivent faire parvenir une requisition au service de personnel de la même façon qu'une demande de matériel est adressée au service d'achats. Ce qui ne veut pas signifier qu'un directeur de personnei puisse obliger un contremaître à accepter n'importe qui. Car c'est la fonction du directeur de personnel de découvrir les sujets de choix que l'usine aura intérêt à accueillir, comme c'est celle du service d'achats de procurer à l'usine de bons approvisionnements. Il est important de souligner, toutefois, que l'embauchage au hasard par le contremaitre ne devrait pas être permis. J'ai fréquemment observé que des contremaitres accordaient leur préférence aux parents et amis, etc., alors qu'en règle générale on doit embaucher que des hommes pouvant servir efficacement.

Dans l'éventualité d'un congédiement, le surintendant ou le contremaitre concerné avisera le directeur de personnel que les services d'un employé désigné ne sont plus requis. Il appartient alors au directeur de personnel de contrôler si aucune injustice n'est faite à cet employé en le congédiant. Il y a des lois en notre pays qui prescrivent ce qui doit être fait en de telles circonstances, et le directeur du personnel doit en tenir compte.

Toute augmentation de salaire doit être présentée sous la forme d'une recommandation au directeur de personnel, qui à son tour, s'il y accède, la fera confirmer par le président du conseil d'administration. A mon avis, toute recommandation en vue d'une augmentation du salaire d'un employé individuel devrait être examinée à fond afin de déterminer en premier lieu si cette augmentation est justifiée et ensuite, si elle, correspond à la politique de la compagnie, ou si elle contrevient au taux payé à des employés exécutant un travail identique. Je crois fermement qu'en cette matière, les contremaitres ne devraient pas avoir le dernier mot.

Le directeur de personnel, lors d'une demande de nouveaux employés, doit s'efforcer' de compléter les cadres à même ceux qui sont déjà au service de l'établissement, plutôt que d'engager sans discernement. Il agit alors comme le pourvoyeur qui cherche à satisfaire aux demandes de matériel d'après ce qu'il a en stock plutôt 


\section{PERSONNEL}

que d'acheter d'autres marchandises. En plusieurs occasions, le directeur de personnel, s'il en fait l'essai, réussira à transterer un employé à un autre département et combler ansi la vacance sans ajouter à la iiste de paie.

\section{Relations avec les syndicats}

Les relations avec les unions ouvrières doivent être faites par l intermediaire du directeur de personnel. Let ofticier do.t connaitre parfaitement les lois et règlements relàtifs au travall. Son role l'oblige aussi à determiner ce qui ne doit pas faire l'objet des conventions collectives cie travari. Lans tous les cas, l'officier de placement doit s'occuper des relations avec les chefs ouvriers qu'il doit s appliquer à connaitre personnellement afin de les convancre de ses intentions droites ainsi que de son désir de traiter avec eux et de s'en tenir aux conventions signées.

Le directeur de personnel ievrait régler tous les griefs; plus il en élıminera, moins nombreuses seront les difficultés avec les ouvriers. Constamment attentif, il devra s'efforcer d'ajuster et d'éliminer les griefs avant qu'ils rie. s'aggravent.

\section{Services médicaux}

L'engagement du personnel soulève une multitude de problèmes dont l'établissement d'un service médical à l'usine. Si le surintendant de personnel veut remplir efficacement sa fonction, il doit s'en occuper lorsqu'un tel service fonctionne déjà. . Il est important afin que la compagnie n'emploie pas de personnel souffrant de maladies. contagieuses ou de déficiences pouvant conduire à des accidents industriels. Le directeur de personnel doit se plier à ces exigences. S'il y a un plan de pension de retraite, il doit apporter une attention particulière à l'âge et à l'état physique de l'employé.

\section{Services de prévention}

I1 faut aussi considérer l'établissement de services sécuritaires. A mon avis, l'officier de sécurité à l'usine doit relever du directeur de personnel à qui il appartient d'élaborer les règlements du service de sécurité et de voir à ce que les assemblées de ce service soient tenues régulièrement. Il doit aussi y assister et prendre connaissance des procès-verbaux des réunions. Il lui revient, en outre, d'établir les règlements sécuritaires et, ce qui est important, de s'en faire le propagandiste. Il doit mettre tout le soin nécessaire à la diffusion des idées de sécurité à l'usine et s'occuper de la tenue des rapports relatifs à l'expérience accidentelle.

\section{Régime d'apprentissage}

Afin que l'entreprise ait à sa disposition une réserve constante de main-d'oeuvre qualifiée, le directeur de per- sonnel doit être responsable de l'exécution d'un programme dentrainement. Il doit rédiger à cette fin, un cours de formation professionnelle prévoyant un entrainement methodique et s'assurer qu'un nombre suffisant d'apprentis passent par tous les stages prévus. C'est également une pratique qui s'est avéree très avantageuse que celle d'embaucher des étudiants durant leurs vacances d'été. Le directeur de personnel doit se tenir en étroite relation avec les écoles de métiers et les universités en vue de rechercher les étudiants désireux d'offrir leurs services à i'entreprise au cours de leurs vacances et de lui procurer ainsi un personnel de choix.

\section{Bien-être et cantines}

Les activités reiatives au bicn-être des employés, teiles celles se rapportant aux associations de secours mutuel, aux bénéfices en maladie, au plan d'assurance-vie, etc., et aux cantines ne doivent pas laisser indifférent le dir ecteur de personnel. Bien plus, il doit coordonner ces activités et favoriser l'étude, dans les cercles d'employés, des problèmes relatifs à leur bien-être. C'est un grand avantage pour une entreprise que d'être assurée que quelqu'un s'occupe du bien-être de son personnel. Dans une communauté humaine il y a toujours quelqu'un qui requiert de l'aide dans ses difficultés, le directeur de personnel doit pouvoir assurer ce service. Il sera facilité par l'élaboration d'une organisation commune pour tous les travailleurs selon la formule coopérative. Les cantines, en raison de leur grande utilité pour le personnel, exigent une surveillance étroite, et les profits réalisés, si possible, doivent être versés à un fonds de secours.

J'ai déjà souligné la nécessité de connaitre les coûts de production. En autant qu'il sera possible, le directeur de personnel devrait avoir eu l'avantage de passer par tous les rouages de l'entreprise et posséder une connaissance précise des diverses fonctions productives. Quoiqu'il en soit, il est indispensable qu'il étudie les diverses occupations. Il importe qu'il connaisse la technique de l'évaluation des tâches afin de lui permettre de parler de celles-ci intelligemment. La question de l'«étude des temps » est aussi essentielle. Je crois que dans plusieurs maisons d'éducation on a complètement négligé d'y attacher de l'importance. Pourtant, elle est maintenant une question de premier plan dans l'industrie et un directeur de personnel ne doit pas l'ignorer. Il peut en être question dans' la politique d'établissement des taux de salaire et dans les relations avec les unions ouvrières.

Enfin, j'attire votre attention sur la nécessité de contacts quotidiens à l'usine. La place du directeur de personnel n'est pas en face d'un bureau. Il doit être constamment à l'usine, en relations avec les occupations et le personnel; il doit entretenir de fréquentes conversations avec les contremaitres sur leurs besoins. De cette façon, les griefs lui seront formulés et il pourra prendre action. A mon avis, plusieurs de ces griefs sont justifiés; d'autres s'appuient sur des informations fausses qu'il peut alors rectifier. Le directeur de personnel doit, de toute nécessité, prendre le temps de considérer et de régler les griefs. Si une entreprise vient à laisser au chef ouvrier le soin de lui faire des recommandations en vue de l'inciter à mettre ordre à cet état de choses, alors qu'elle peut autrement y remédier, c'est avouer que le directeur de personnel a failli à sa tâche.

T. Roger McLagan 